\title{
Functional and Structural Changes in End-Stage Kidney Disease due to Glomerulonephritis Induced by the Recombinant $\alpha 3$ (IV)NC1 Domain
}

\author{
Seiji NISHIBAYASHI ${ }^{1,4)}$, Katsuji HATTORI ${ }^{2)}$, Takahiro HIRANO ${ }^{2)}$, Kenji UEHARA ${ }^{2)}$, \\ Yoshimasa NAKANO ${ }^{2)}$, Miki AIHARA ${ }^{2)}$, Yoshihisa YAMADA ${ }^{2)}$, \\ Masahiro MURAGUCHI ${ }^{3)}$, Fusako IWATA ${ }^{3)}$, and Yoshiharu TAKIGUCHI ${ }^{4)}$ \\ ${ }^{1)}$ Quests Research Institute, ${ }^{2)}$ First Institute of New Drug Discovery, ${ }^{3)}$ Institute of Biomedical Innovation, \\ Otsuka Pharmaceutical Co., Ltd., 463-10 Kagasuno, Kawauchi, Tokushima 771-0192, and \\ ${ }^{4)}$ Department of Clinical pharmacology, Institute of Health Bioscience, The University of \\ Tokushima Graduate School, 1-78-1 Shomachi, Tokushima 770-8505, Japan
}

\begin{abstract}
The aim of this study was to develop and characterize a rat glomerulonephritis model, which progresses to renal fibrosis and renal failure. A single immunization of female WKY rats with more than $10 \mu \mathrm{g}$ of recombinant $\alpha 3(\mathrm{IV}) \mathrm{NC} 1$ protein caused severe proteinuria followed by progressive increases in plasma creatinine and blood urea nitrogen (BUN) level within 42 days. Sequential histopathological evaluation revealed crescent formation in glomeruli followed by tubular dilation and interstitial fibrosis. Hydroxyproline content and expression of type I collagen and $\alpha$ smooth muscle actin genes in the renal cortex increased as renal dysfunction progressed. Furthermore, the TGF- $\beta 1$ level in the renal cortex also increased. In the evaluation of antinephritic agents in this model, prednisolone and mycophenolate mofetil (MMF) treatment significantly decreased plasma creatinine and BUN, and suppressed renal fibrosis and histological changes involving crescent formation, compared with the vehicle-treated nephritic rats, whereas lisinopril treatment failed to improve renal function and histology. We demonstrated that immunization of female WKY rats with a sufficient dose of recombinant $\alpha 3$ (IV)NC1 induces end-stage kidney disease accompanied by renal fibrosis. The relatively short period needed to induce the disease and the high incidence of functional and structural changes were considered a great advantage of this model for clarifying the mechanisms of progressive glomerulonephritis and for evaluating agents used to treat renal failure.
\end{abstract}

Key words: $\alpha 3$ chain of type IV collagen, end-stage renal failure, renal fibrosis

\section{Introduction}

Glomerular injury is the major cause of glomerulonephritis that leads to end-stage renal disease (ESRD).
Furthermore, immunologically induced inflammatory injury to the glomeruli and severe glomerulosclerosis are characteristic features of human glomerulonephritis [18]. Recently, evidence has been increasing to support

(Received 13 September 2009 / Accepted 26 October 2009)

Address corresponding: S. Nishibayashi, Quests Research Institute, Otsuka Pharmaceutical Co., Ltd., 463-10 Kagasuno, Kawauchi, Tokushima 771-0192, Japan 
a role for cell-mediated immunity, including autoreactive T lymphocytes and macrophages, in the pathogenesis of glomerulonephritis [5, 27]. However, the immunological reaction and associated pathway alone cannot fully explain many aspects of the progression of human glomerulonephritis to ESRD. To elucidate the pathological mechanisms of the progression to ESRD, it is essential to establish appropriate disease models that express these features.

The $\alpha 3$ chain of type IV collagen is a component of the glomerular basement membrane (GBM). The noncollagenous domain of $\alpha 3$ chain of type IV collagen [ $\alpha 3(\mathrm{IV}) \mathrm{NC} 1]$, which has been localized to the amino terminal of the molecule, has been identified as the major epitope of the autoantigen in Goodpasture syndrome $[4,15,24]$, an autoimmune disorder characterized by rapidly progressive glomerulonephritis and lung hemorrhage caused by autoantibodies to GBM and alveoli [25, 28].

Some rodent glomerulonephritis models reported in previous studies of susceptible mouse and rat strains have been induced by injection of anti- $\alpha 3$ (IV)NC1 antiserum or active immunization with $\alpha 3$ (IV)NC1 purified from heterologous GBM, or with recombinant $\alpha 3$ (IV) $\mathrm{NC} 1$ protein $[8,11,23]$. In particular, severe proliferative and necrotizing glomerulonephritis can be induced in the WKY rat, but not other rat strains [13, 19, 22]. In these models, proteinuria, hematuria, and induction of inflammatory injury to the glomeruli, including deposition of antibodies directed against components of GBM or immune complexes, are commonly observed $[1,20]$. However, manifestations of these glomerulonephritis models were variously dependent on the epitope and amount of injected antibody and the factors that cause the worsening of renal function remain unclear. Therefore, in this study, we obtained rat recombinant $\alpha 3$ (IV) $\mathrm{NC} 1$ protein that had been expressed in mammalian cells, and investigated whether progressive renal dysfunction and sclerotic lesions could be developed in female WKY rats by immunization with this $\alpha 3$ (IV)NC1 protein. In addition, we characterized this model by evaluating the efficacy of certain anti-nephritic drugs, namely prednisolone, mycophenolate mofetil (MMF) and lisinopril.

\section{Materials and Methods}

\section{Experimental animals}

Female WKY rats (Charles River Japan Inc., Yokohama, Japan) were purchased at six weeks of age and housed until eight weeks of age. Rats were allowed free access to chow and tap water. All experiments were performed under the Institutional Guidelines for Animal Experimentation (Otsuka Pharmaceutical, Tokushima, Japan).

\section{Preparation of recombinant rat $\alpha 3(I V) N C 1$ protein}

The cDNA fragment encoding the rat collagen $\alpha 3$ (IV) $\mathrm{NC} 1$ domain was obtained by reverse-transcription PCR cloning of total RNA from the rat kidney using a pair of primers based on the published rat sequence [21]. A cDNA fragment was subcloned into a pT7 shuttle vector (Invitrogen Corp., Carlsbad, CA, USA) and sequenced using a CEQ2000 DNA Analysis System (Beckman Coulter, Fullerton, CA, USA). The cDNA fragment was inserted into a pSecTag2 expression vector encoding the Zeocin resistance gene and histidine hexamer (Invitrogen). The expression vectors were transfected into CHOK1 cells (DS Pharma Biomedical, Osaka, Japan) using Lipofectamine 2000 (Invitrogen). Cell lines secreting recombinant $\alpha 3(\mathrm{IV}) \mathrm{NC} 1$ protein into the culture medium were selected by adding $50 \mu \mathrm{g} / \mathrm{ml}$ Zeocin (Invitrogen) to Dulbecco's modified Eagle medium containing 10\% fetal bovine serum and $0.1 \%$ penicillin/streptomycin. The production of recombinant $\alpha 3$ (IV)NC1 protein in the medium was quantified by ELISA using anti-histidine tag antibodies (Invitrogen). To purify the recombinant $\alpha 3$ (IV)NC1 protein, the culture medium was collected and run over a histidine-binding column and the bound protein was eluted with $1 \mathrm{M}$ imidazole. After dialysis with $50 \mathrm{mM}$ Tris buffer (pH 8.0) containing $0.5 \mathrm{M} \mathrm{NaCl}$, the protein was concentrated using Amicon concentrators (Millipore Corporation, Billerica, MA, USA) and stored at $-80^{\circ} \mathrm{C}$ until use. The purified protein was analyzed by sodium dodecyl sulfate polyacrylamide gel electrophoresis (SDS-PAGE) and western blotting using antihistidine tag antibodies. 
Examination of renal function, antibodies, and systolic blood pressure

Blood was collected from the tail vein and plasma was obtained after centrifugation at 3,000 rpm for $15 \mathrm{~min}$ at $4^{\circ} \mathrm{C}$. After collecting blood, the animals were kept in individual metabolic cages for $16 \mathrm{~h}$; urine samples were then collected and centrifuged at 3,000 rpm for $15 \mathrm{~min}$ at $4^{\circ} \mathrm{C}$. Plasma and urinary creatinine concentrations were measured by the creatininase-HMMPS method [12]. Plasma blood urea nitrogen (BUN) concentrations were measured by the urease-GIDH method [6] (Wako Pure Chemicals, Osaka, Japan). Urinary protein concentrations were measured by the pyrogallol red method (Wako Pure Chemicals). Creatinine, BUN, and urinary protein were measured using a Corbas Mira autoanalysis system (Roche Diagnostic, Basel, Switzerland). Circulating anti- $\alpha 3$ (IV)NC1 antibodies were measured in plasma by a solid-phase enzyme-linked immunosorbent assay (ELISA). Purified recombinant $\alpha 3$ (IV)NC1 was coated at a concentration of $1 \mu \mathrm{g} / \mathrm{ml}$ onto a microtiter plate (Gibco, Paisley, UK) by overnight incubation at $4^{\circ} \mathrm{C}$ then plasma was applied for $1 \mathrm{~h}$ at $37^{\circ} \mathrm{C}$. Bound anti- $\alpha 3$ (IV)NC1 antibody was detected by horseradish peroxidase-conjugated sheep anti-IgG (Sigma-Aldrich Corporation, St. Louis, MS, USA) and developed using the substrate ortho-phenylenediamine dihydrochloride (OPD, Sigma-Aldrich Corporation).The absorbance of each well was measured at $492 \mathrm{~nm}$ and the results expressed as the mean OD of triplicate wells.

The systolic blood pressure (SBP) was measured in conscious rats by the tail-cuff method with an automatic sphygmomanometer (BP98A; Softron, Tokyo, Japan).

Assessment of collagen accumulation and TFG- $\beta$ expression in the renal cortex

Renal cortices were collected for analysis of renal fibrosis and stored at $-80^{\circ} \mathrm{C}$ until use. Renal collagen content was assessed as the amount of hydroxyproline by a modified method described by Stegemann and Stalder [26]. Briefly, $100 \mathrm{mg}$ pieces of wet renal cortex tissue were homogenized in saline and the homogenate was hydrolyzed in $6 \mathrm{~N} \mathrm{HCl}$ by incubation at $110^{\circ} \mathrm{C}$ overnight. Hydrolysates were neutralized with $6 \mathrm{~N} \mathrm{KOH}$, oxidized by chloramine $\mathrm{T}$, then mixed with p-dimethylaminobenzaldehyde. The sample was extracted with toluene and the absorbance was measured at $560 \mathrm{~nm}$ after the toluene fraction had reacted with Ehrlich's reagent. Renal transforming growth factor (TGF)- $\beta 1$ concentration was measured using a commercially available ELISA kit specific to rat TGF- $\beta 1$ (Emax ImmunoAssay system, Promega, Madison, WI, USA).

\section{Measurement of mRNA expression by real-time quantitative} reverse transcription $P C R$

Renal cortices for real-time PCR analysis were collected in RNA-later solution (Qiagen, Valencia, CA, USA) and total RNA was extracted and purified using a RNeasy mini kit and a RNeasy mini Elute Cleanup Kit (Qiagen). cDNA synthesis was performed using a Superscript TM first strand Synthesis System (Invitrogen). Real-time reverse transcription- PCR was performed on an ABI Prism 7000 Sequence Detection System (Applied Biosystems, Foster city, CA, USA). Amplified gene expression was normalized by $\beta 2$-microglobulin gene expression as an internal control and data were expressed as value relative to the gene expression levels in the normal rat. The sequences of specific oligonucleotide primers and FAM (5')- and TAMRA (3')-labeled hybridization probes are outlined in Table 1.

\section{Renal histopathology}

Renal tissue for histological examination was fixed in $10 \%$ neutral-buffered formaldehyde solution, embedded in paraffin, sectioned into 3-4- $\mu \mathrm{m}$-thick slices and stained with Periodic acid-Schiff (PAS) reagent and hematoxylin or Masson's trichrome (MT). Glomerular and tubular lesions were assessed in the PAS-hematoxylin stained sections. Severity of glomerular and tubular lesions including crescent formation, tubular dilation and inflammation were graded on a scale from 0 to 4 ( 0 : No abnormality, 1: Minimal, 2: Mild, 3: Moderate, 4: Marked abnormality). Cross-sections of fifty glomeruli from each rat were evaluated and the average score was calculated. All morphologic evaluations were performed by a renal pathologist who was unaware of the source of the renal tissue.

\section{Disease induction and experimental design}

Three separate experiments were performed. 
Table 1. Primers and probes for RT-PCR amplification of cDNA fragment

\begin{tabular}{|c|c|}
\hline Gene names & Primer and probe sequences ( 5 'to 3 ') \\
\hline $\begin{array}{l}\text { Rat type I collagen } \alpha 1 \\
(\mathrm{COL} 1 \alpha 1)\end{array}$ & $\begin{array}{l}\text { Forward primer: GCAACATGGAGACAGGTCAG } \\
\text { Reverse primer: GAATCCATCGGTCATGCTCT } \\
\text { TaqMan probe: CTCAGAAGAACTGGTACATCAGCCCAAACC }\end{array}$ \\
\hline $\begin{array}{l}\text { Rat } \alpha \text {-smooth muscle actin } \\
(\alpha \text { SMA) }\end{array}$ & $\begin{array}{l}\text { Forward primer: CCCGCTCTGTCTCTAGCAC } \\
\text { Reverse primer: GAAGGGTAGCACATG TCTGATTT } \\
\text { TaqMan probe: TTTGTGGATCAGCGCCTTCAGTTCT TT }\end{array}$ \\
\hline $\begin{array}{l}\text { Rat connective tissue growth factor } \\
\text { (CTGF) }\end{array}$ & $\begin{array}{l}\text { Forward primer: GTGAGCCTCCTGGAAGAGTG } \\
\text { Reverse primer: CAGGTCTGTC TGCAAGCAAG } \\
\text { TaqMan probe: TGAGGTCATTTTAAAAGCAGCGATATGCCT }\end{array}$ \\
\hline $\begin{array}{l}\text { Rat } \beta 2 \text {-microgloburin } \\
(\beta 2 \mathrm{M})\end{array}$ & $\begin{array}{l}\text { Forward primer: ACCGA GACCG ATGTA TATGC } \\
\text { Reverse primer: TGATT CAGAG CTCCA TAGAG } \\
\text { TaqMan probe: AGAGT TAAAC ACGTC ACTCT GAAGG AGCCC }\end{array}$ \\
\hline
\end{tabular}

Experimental protocol 1: Determination of dose of $r \mathrm{NC1}$ antigen required to induce rapidly progressive glomerulonephritis

Three groups of six rats were immunized intradermally in the footpads and back with 3,10 , or $20 \mu \mathrm{g}$ of ro3(IV)NC1 in $500 \mu 1$ PBS emulsified in an equal volume of complete Freund's adjuvant (CFA, Difco Laboratory, Detroit, MI, USA). Blood and urine collection were performed at 42 days after immunization and proteinuria, plasma creatinine and BUN were measured. Kidneys were collected at sacrifice and processed to assess interstitial collagen accumulation and histopathology.

Experimental protocol 2: Characterization of glomerulonephritis during the course of renal failure

Thirty rats were immunized with $20 \mu \mathrm{g}$ of ra3(IV) NC1 in $500 \mu 1$ PBS emulsified in an equal volume of CFA and divided into five groups. After urine collection, blood and kidneys were collected at 7, 14, 21, 28, and 42 days after immunization. The kidneys were processed to assess interstitial collagen accumulation, gene expression related to tissue fibrosis by real-time PCR, renal TGF- $\beta$ content and histopathology.

Experimental protocol 3: Evaluation of antinephritic agents in the prevention of progression of renal failure

Twenty-eight rats were immunized with $20 \mu \mathrm{g}$ of ra3(IV)NC1 in $500 \mu 1$ PBS emulsified in an equal volume of CFA. Fourteen days after immunization, the rats were divided into four groups, including the normal rat group; all groups had seven animals. Three groups of rats were orally given daily prednisolone (Wako) at a dose of $2 \mathrm{mg} / \mathrm{kg}$ body weight, MMF (Cellcept, Roche, Basel, Switzerland) at a dose of $20 \mathrm{mg} / \mathrm{kg}$ or lisinopril (Wako) at a dose of $10 \mathrm{mg} / \mathrm{kg}$. Every day, control and normal rats were given the same volume of $5 \%$ arabic gum solution as a vehicle. Blood and urine collection and SBP measurement were performed at 14, 28, and 35 days after immunization. At the end point of the study (Day 35), blood and kidneys were collected at sacrifice and processed for evaluation of treatment efficacy.

\section{Statistical analysis}

Data were expressed as the mean $\pm \mathrm{SD}$. The significance of differences between groups was evaluated by Student's $t$-test and analysis of variance using the SAS system (SAS Institute, Cary, NC, USA). $P<0.05$ was considered statistically significant.

\section{Results}

\section{Characterization of recombinant rat $\alpha 3(I V) N C 1$ protein}

The rat $\alpha 3$ (IV)NC1 protein was secreted into the supernatant of stably transfected $\mathrm{CHO}-\mathrm{K} 1$ cells as a recombinant fusion protein with a histidine-tag. This recombinant protein consisted of 230 amino acids and contained the rat type IV collagen $\alpha 3$ non-collagenous domain which has been identified as the Goodpasture antigen. SDS-PAGE analysis identified r $\alpha 3$ (IV)NC1 protein as a 
$\mathbf{A}$

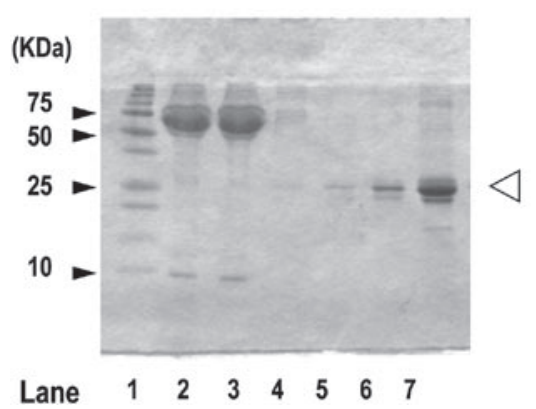

B

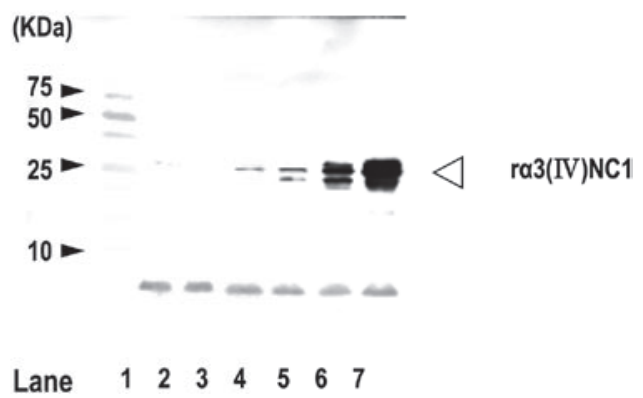

Fig. 1. Characterization of r $\alpha 3(\mathrm{IV}) \mathrm{NC} 1$ domains. SDS-PAGE analysis, staining with Coomassie brilliant blue (A). Western blot analysis with anti-His tag antibodies (B). Lane 1: Molecular mass indicator; Lane 2: Culture medium; Lane 3: Flow-through fraction; Lane 4: Washed fraction with $50 \mathrm{mM}$ imidazole; Lanes 5 and 6: Fractions eluted with $1 \mathrm{M}$ imidazole; Lane 7: Purified protein.

Table 2. Urinary protein excetion, plasma creatinine, and BUN level in WKY rats immunized with recombinant rat $\alpha 3(\mathrm{IV}) \mathrm{NC} 1$ and $\mathrm{CFA}$

\begin{tabular}{lccc}
\hline & $\begin{array}{c}\text { Urinary protein excretion } \\
(\mathrm{mg} / 16 \mathrm{~h})\end{array}$ & $\begin{array}{c}\text { Plasma creatinine } \\
(\mathrm{mg} / \mathrm{dl})\end{array}$ & $\begin{array}{c}\mathrm{BUN} \\
(\mathrm{mg} / \mathrm{dl})\end{array}$ \\
\hline $3 \mu \mathrm{g}$ of $\mathrm{r \alpha 3}(\mathrm{IV}) \mathrm{NC} 1$ & $63.1 \pm 27.0$ & $0.49 \pm 0.05$ & $16.8 \pm 2.3$ \\
$10 \mu \mathrm{g}$ of $\mathrm{r \alpha 3}(\mathrm{IV}) \mathrm{NC} 1$ & $85.5 \pm 10.2^{* *}$ & $2.24 \pm 0.22^{*}$ & $133.9 \pm 6.4^{* *}$ \\
$20 \mu \mathrm{g}$ of $\mathrm{r} \alpha 3(\mathrm{IV}) \mathrm{NC} 1$ & $67.7 \pm 6.4^{* *}$ & $2.00 \pm 0.18^{*}$ & $102.4 \pm 10.2^{*}$ \\
$\mathrm{CFA}$ alone & $7.6 \pm 1.4$ & $0.52 \pm 0.01$ & $14.3 \pm 0.1$ \\
\hline
\end{tabular}

Data are represented as mean \pm SD at 42 days after immunization. $* P<0.05$, $* * P<0.01$; comparison with the CFA-immunized control rats was performed by Student's $t$-test.

band of molecular mass $24 \mathrm{kDa}$ that was eluted with 1 $\mathrm{M}$ imidazole and purified (Fig. 1A, lanes 5 to 7 ). Western blot analysis also showed that ra3(IV)NC1 protein could be detected as a monomeric isoform at the predicted molecular weight of approximately $24 \mathrm{kDa}$ by anti-histidine-tag antibody (Fig. 1B, lanes 5 to 7 ).

\section{Recombinant NCl protein induced severe glomerulonephritis in WKY rats}

WKY rats were immunized with 3, 10, or $20 \mu \mathrm{g}$ of ro3(IV)NC1 protein in emulsified CFA. As shown in Table 2, rats immunized with $3 \mu \mathrm{g}$ of ro3(IV)NC1 protein developed proteinuria, while plasma creatinine and BUN levels remained almost within the normal range until Day 42. The body weight of these rats continuously increased, similar to CFA-immunized control rats, until the end of study (Fig. 2). The rats immunized with 10 or $20 \mu \mathrm{g}$ of ra3(IV)NC1 protein also developed pro-

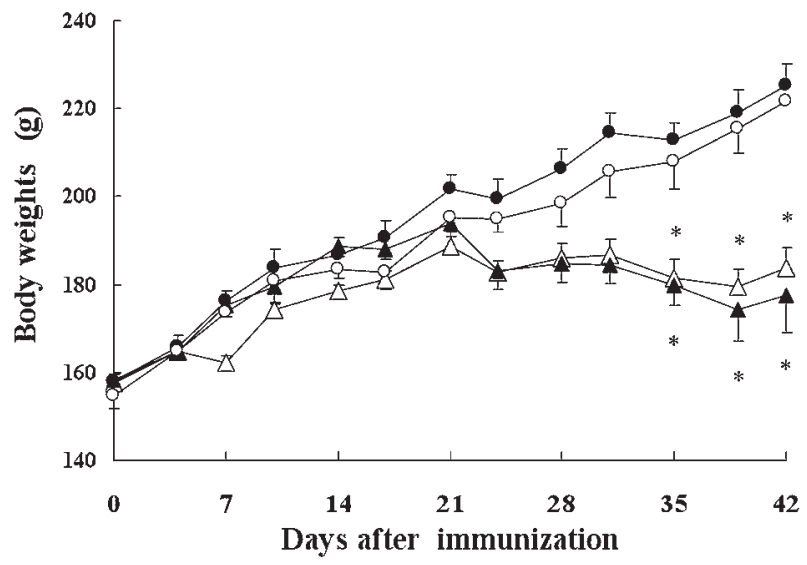

Fig. 2. Body weights of WKY rats immunized with recombinant rat $\alpha 3$ (IV)NC1 and CFA. $3 \mu \mathrm{g}$ of r $\alpha 3$ (IV)NC1, $\boldsymbol{\Delta}: 10$ $\mu \mathrm{g}$ of $\mathrm{r} \alpha 3(\mathrm{IV}) \mathrm{NC} 1, \triangle: 20 \mu \mathrm{g}$ of $\mathrm{r} \alpha 3(\mathrm{IV}) \mathrm{NC} 1, \bigcirc:$ CFA alone. Data are represented as mean $\pm \mathrm{SD}$ at Day 42 after immunization. $* P<0.05$; comparison with the CFA-immunized control rats was performed by Student's $t$-test. 
A

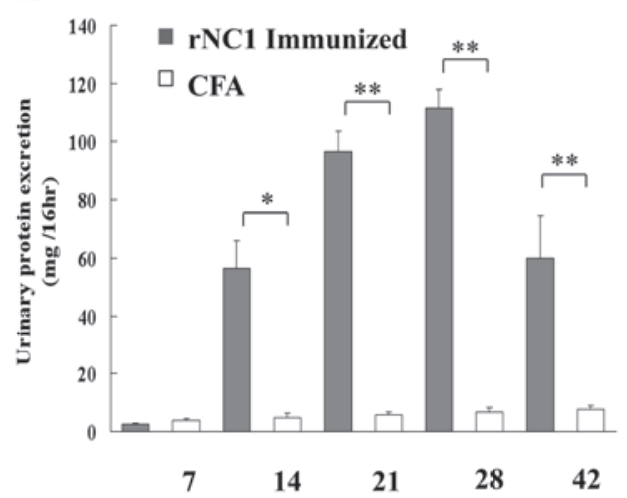

C

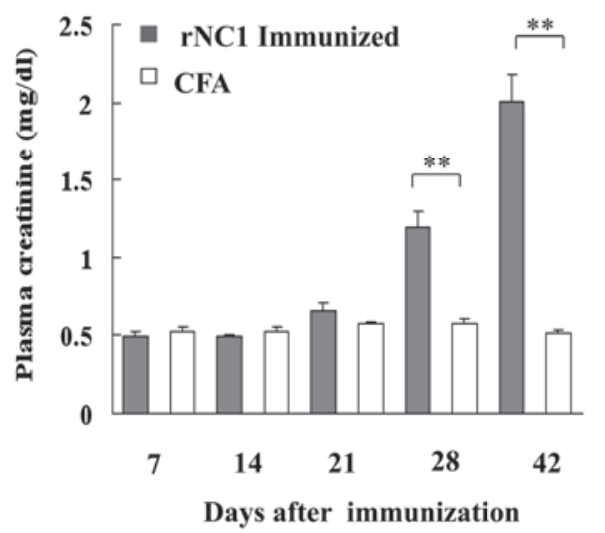

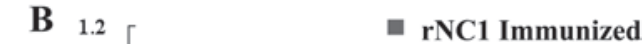

$\square$ CFA

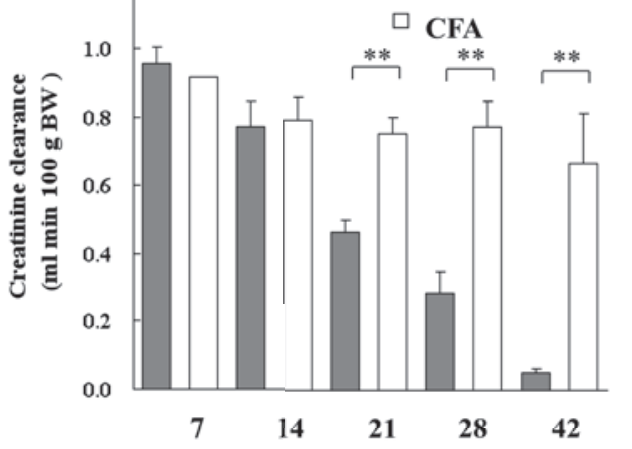

Days after immunization

D

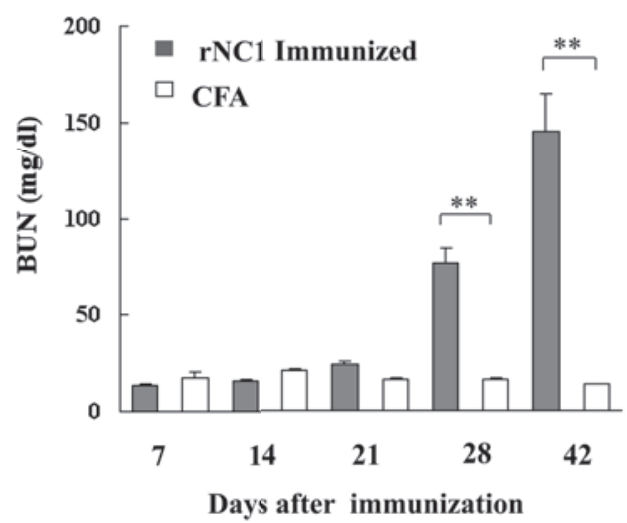

Fig. 3. Kinetics of urinary protein excretion (A), creatinine clearance (B), plasma creatinine level (C), and BUN level (D) in WKY rats immunized with $20 \mu \mathrm{g}$ of $\alpha 3$ (IV)NC1. Data are represented as mean \pm SD at Day 42 after immunization. $* P<0.05, * * P<0.01$; comparison with the CFA-immunized control rats was performed by Student's $t$-test.

teinuria at Day 42. In these rats, plasma creatinine and BUN levels were significantly increased compared with CFA-immunized control rats at Day 42 (Table 2). These rNC1 protein-treated rats also showed a significant decrease in body weight at Day 35 compared with CFAimmunized control rats and this continued to decrease until Day 42 in accordance with renal function deterioration (Fig. 2). These results indicate that ro3(IV)NC1 antigen immunization of WKY rats induces renal dysfunction and that the development of progressive renal failure in WKY rats requires immunization with more than $10 \mu \mathrm{g}$ of r $\alpha 3(\mathrm{IV}) \mathrm{NC} 1$ protein.

Time course of renal function and antibodies in rats with glomerulonephritis

To induce renal dysfunction sufficiently, we immunized WKY rats with $20 \mu \mathrm{g}$ of ra3(IV)NC1 protein. In rats immunized with $\mathrm{r} \alpha 3(\mathrm{IV}) \mathrm{NC} 1$ protein, proteinuria was initially detected at Day 14, peaked at Day 28 and then declined until Day 42 after induction of nephritis (Fig. 3A). Creatinine clearance was significantly decreased at Day 21 compared with that of CFA-immunized control rats and continued to decrease until Day 42 after induction of renal dysfunction (Fig. 3B). Plasma creatinine and BUN levels were significantly increased at Day 28 compared with their values in CFA-immunized control rats and continued to rise until Day 42 (Fig. 3C and 3D). Circulating anti- $\alpha 3($ IV)NC1 antibodies were detected at Day 14 and continued to increase until Day 42 in rats immunized with ra3(IV)NC1 protein. CFAimmunized control rats showed no increase in circulating anti- $\alpha 3$ (IV)NC1 antibodies (Fig. 4). 


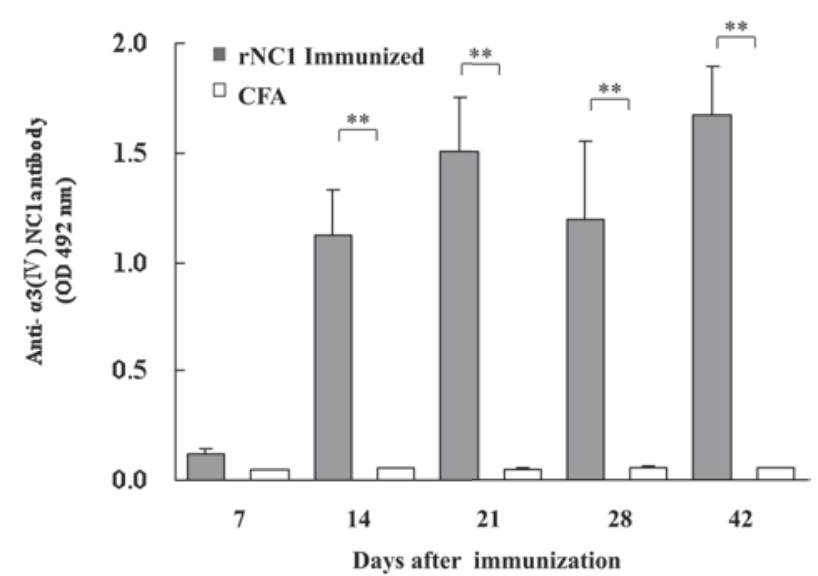

Fig. 4. Circulating anti- $\alpha 3(\mathrm{IV}) \mathrm{NC} 1$ antibodies concentrations in WKY rats immunized with $20 \mu \mathrm{g}$ of $\alpha 3$ (IV)NC1. Data are represented as mean \pm SD at Day 7, 14, 21, 28, and 42 after immunization. $* * P<0.01$; comparison with the CFAimmunized control rats was performed by Student's $t$ test.
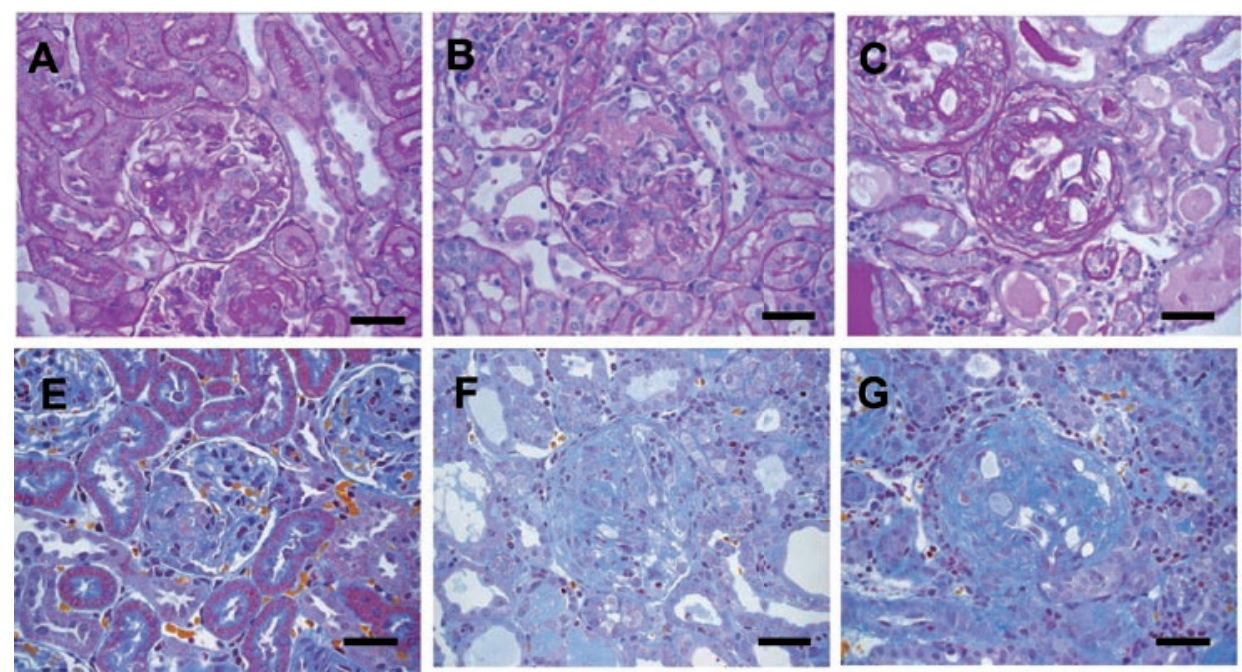
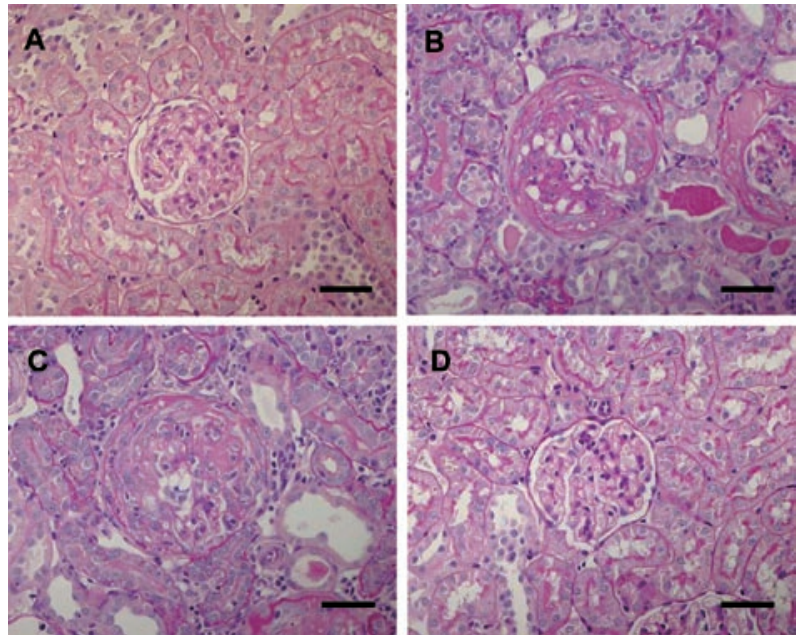

Fig. 5. Representative kidney sections of WKY rats at Day 42 after immunization with $3.0 \mu \mathrm{g}$ (A), $10 \mu \mathrm{g}$ (B), or $20 \mu \mathrm{g}$ (C) of r $\alpha 3$ (IV)NC1 in CFA or CFA alone (D). PAS-hematoxylin staining; Magnification $\times 400$. Bar $=50 \mu \mathrm{m}$.

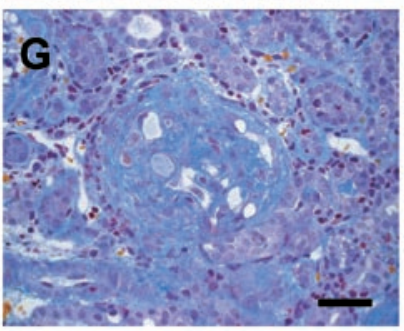

Fig. 6. Representative kidney sections of WKY rats at Day 14 (A, E), Day 28 (B, F), and Day 42 (C, G) after immunization with 20 $\mu \mathrm{g}$ of r $\alpha 3(\mathrm{IV}) \mathrm{NC} 1$ in CFA and at Day 42 after immunization with CFA alone (D, H). Staining; PAS-hematoxylin (A, B, C, D) and $\mathrm{MT}(\mathrm{E}, \mathrm{F}, \mathrm{G}, \mathrm{H})$; Magnification $\times 400$. Bar $=50 \mu \mathrm{m}$.

\section{Glomerular and interstitial histological changes in} progressive renal failure

Histological analysis of kidneys by PAS-hematoxylin staining 42 days after immunization revealed severe crescent formation in rats immunized with 10 and $20 \mu \mathrm{g}$ of ro3(IV)NC1 protein (Fig. 5B and 5C), whereas there were no significant differences in glomerular and tubular architecture in the rats immunized with $3 \mu \mathrm{g}$ of ra3(IV)
$\mathrm{NC} 1$ protein (Fig. 5A) compared with CFA-immunized control rats (Fig. 5D).

In the time-course study, histological analysis using PAS-hematoxylin staining of renal tissue showed thickening of the glomerular basement membrane, tubular dilation and crescent formation in Bowman's capsule at Day 28 , followed by severe crescent formation (Fig. $6 \mathrm{~A}-\mathrm{C})$. After 42 days, obliteration of glomerular capil- 

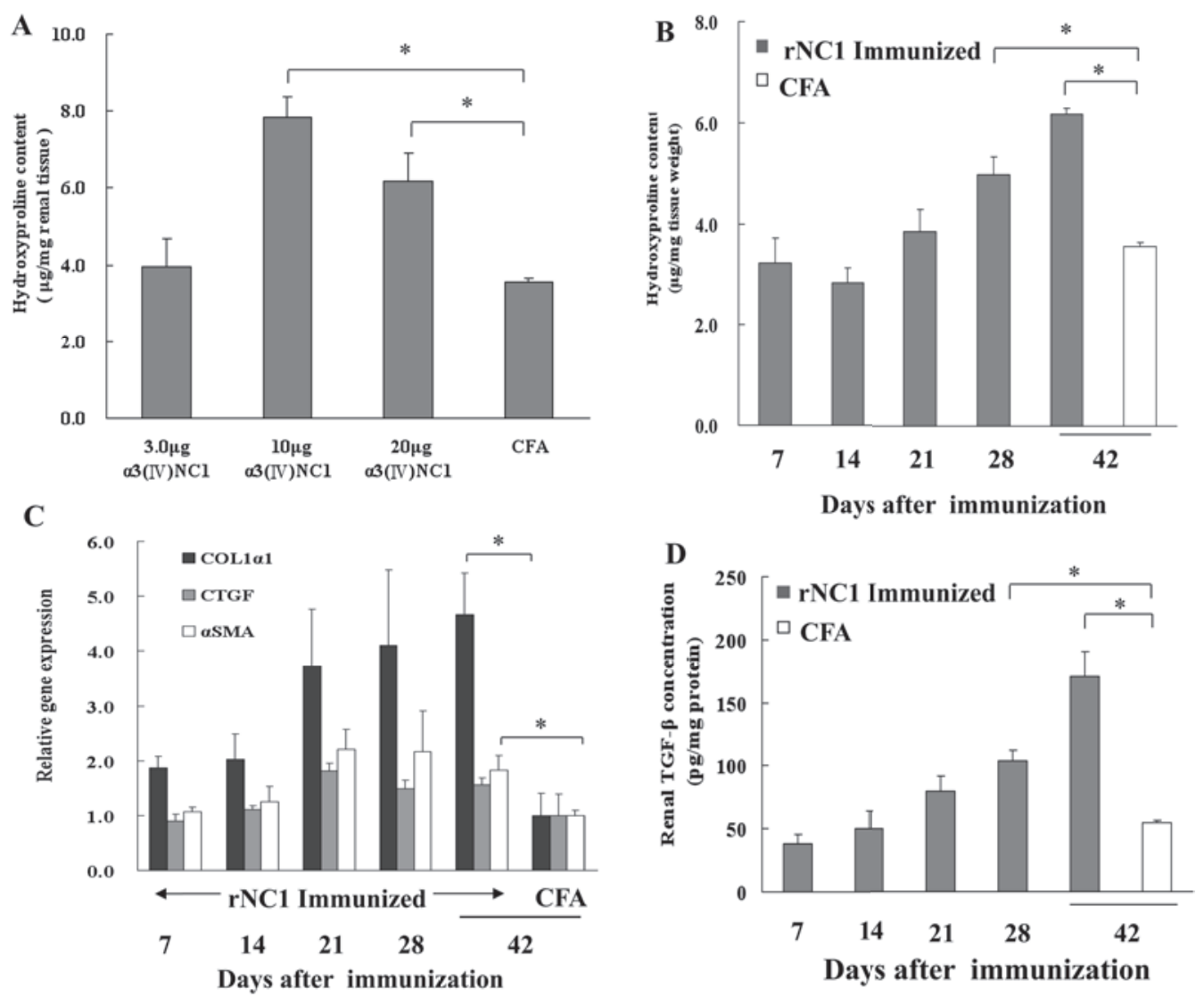

Fig. 7. Hydroxyproline content (A, B), mRNA expression of renal fibrogenic markers (C), and TGF- $\beta$ level (D) of renal cortex in WKY rats immunized with of $\alpha 3$ (IV)NC1. Data are represented as mean \pm SD of each group at Day $7,14,28$, and 42 after immunization. $* P<0.01$; comparison with the CFA-immunized control rats was performed by Student's $t$-test.

laries and cast formation in glomeruli were observed (Fig. 6C).

Histological analysis of MT staining also revealed strong staining of glomeruli compared with CFA-immunized control rats at 28 days (Fig. $6 \mathrm{~F}$ and $6 \mathrm{H}$ ) and interstitial fibrosis that advanced in a time-dependent manner (Fig. 6E-G). We showed that the development of glomerular and interstitial lesions was accompanied by deterioration in renal function.

\section{Progression of renal fibrosis}

In rats immunized with either 10 or $20 \mu \mathrm{g}$ of ra3(IV) $\mathrm{NC} 1$ protein, hydroxyproline content in the renal cortex significantly increased compared with CFA-immunized control rats at Day 42. In contrast, hydroxyproline content was not significantly different in rats immunized with $3 \mu \mathrm{g}$ of ra3(IV)NC1 protein (Fig. 7A).
In the time-course study, hydroxyproline content in the renal cortex started to increase gradually at Day 21 and had doubled by Day 42 compared with CFA-immunized control rats (Fig. 7B).

Real-time PCR analysis showed that type I collagen and $\alpha$ SMA gene expression were upregulated compared with CFA-immunized control rats at Day 21 and these high levels of gene expression were retained until the end of study. However, expression of the CTGE gene, which is also a well-defined tissue fibrosis-related gene, was not significantly different from that in CFA-immunized control rats up to Day 42 (Fig. 7C). Furthermore, ELISA analysis showed that the TGF- $\beta$ level in the renal cortex was significantly increased, compared with CFAimmunized control rats at Day 28, and this high level of expression continued to rise until Day 42 (Fig. 7D). 

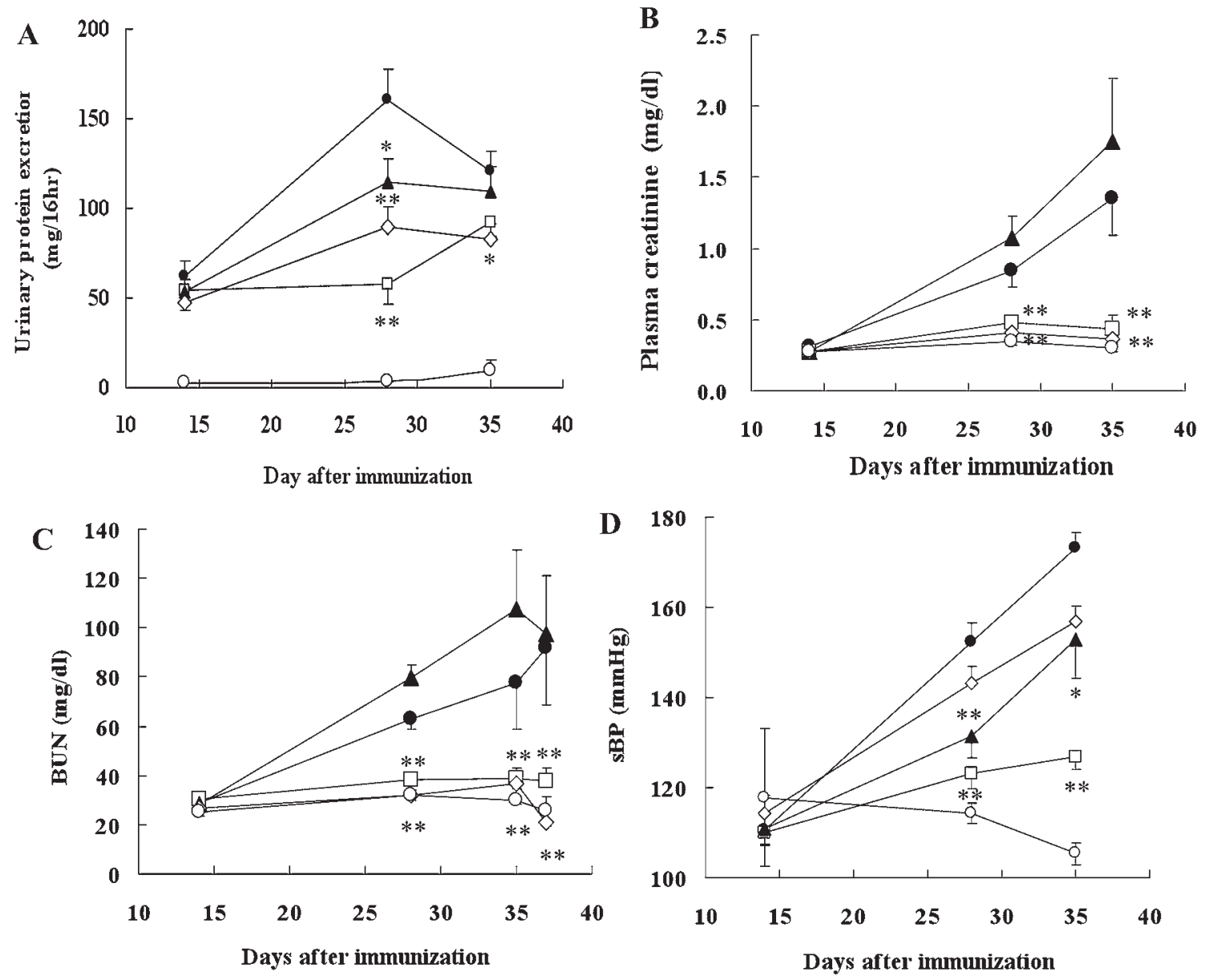

Fig. 8. Effects of prednisolone, MMF, and lisinopril on urinary protein excretion (A), plasma creatinine (B), BUN (C), and systolic blood pressure (D) in WKY rats immunized with of $\alpha 3$ (IV)NC1. Vehicle-treated control rats (O), MMF-treated rats $(\square)$, Lisinopril-treated rats $(\boldsymbol{\Delta})$, Prednisolone-treated rats $(\diamond)$, Normal rats $(\bigcirc)$. Data are represented as mean \pm SD of each group at Day 14, 28, and 35 after immunization with $20 \mu \mathrm{g}$ of ro3(IV)NC1 in CFA. Vehicle or drug was orally administered once a day from Day 14 to 35 . $* P<0.05$, $* * P<0.01$; comparison with the CFA-immunized control rats was performed by Student's $t$-test.

\section{Evaluation of antinephritic agents in rats with glomerulonephritis}

We next examined the characteristics of this model through the evaluation of agents used to treat rapidly progressive glomerulonephritis.

As shown in Fig. 8, administration of MMF or prednisolone significantly prevented proteinuria compared with vehicle-treated control at Day 28 (Fig. 8A). These drugs also significantly prevented plasma creatinine and BUN elevations at Day 28 (Fig. 8B and 8C). Administration of lisinopril also prevented proteinuria and the elevation of systolic blood pressure (Fig. 8A and 8D) but did not prevent the increase in plasma creatinine and
BUN (Fig. 8B and 8C).

Histological analysis showed severe crescentic glomerulonephritis with massive inflammation developed in the vehicle-treated control rats by Day 35 (Fig. 9A). Prednisolone and MMF treatment significantly attenuated crescent formation in glomeruli, inflammation and tubular dilation (Fig. 9B, 9D, and 9F-H), whereas histological evaluation of rats treated with lisinopril showed no difference compared with vehicle-treated controls (Fig. 9C and 9F-H). Moreover, prednisolone and MMF significantly reduced renal fibrosis detected by measurement of the hydroxyproline content at the end of study (Fig. 10). 

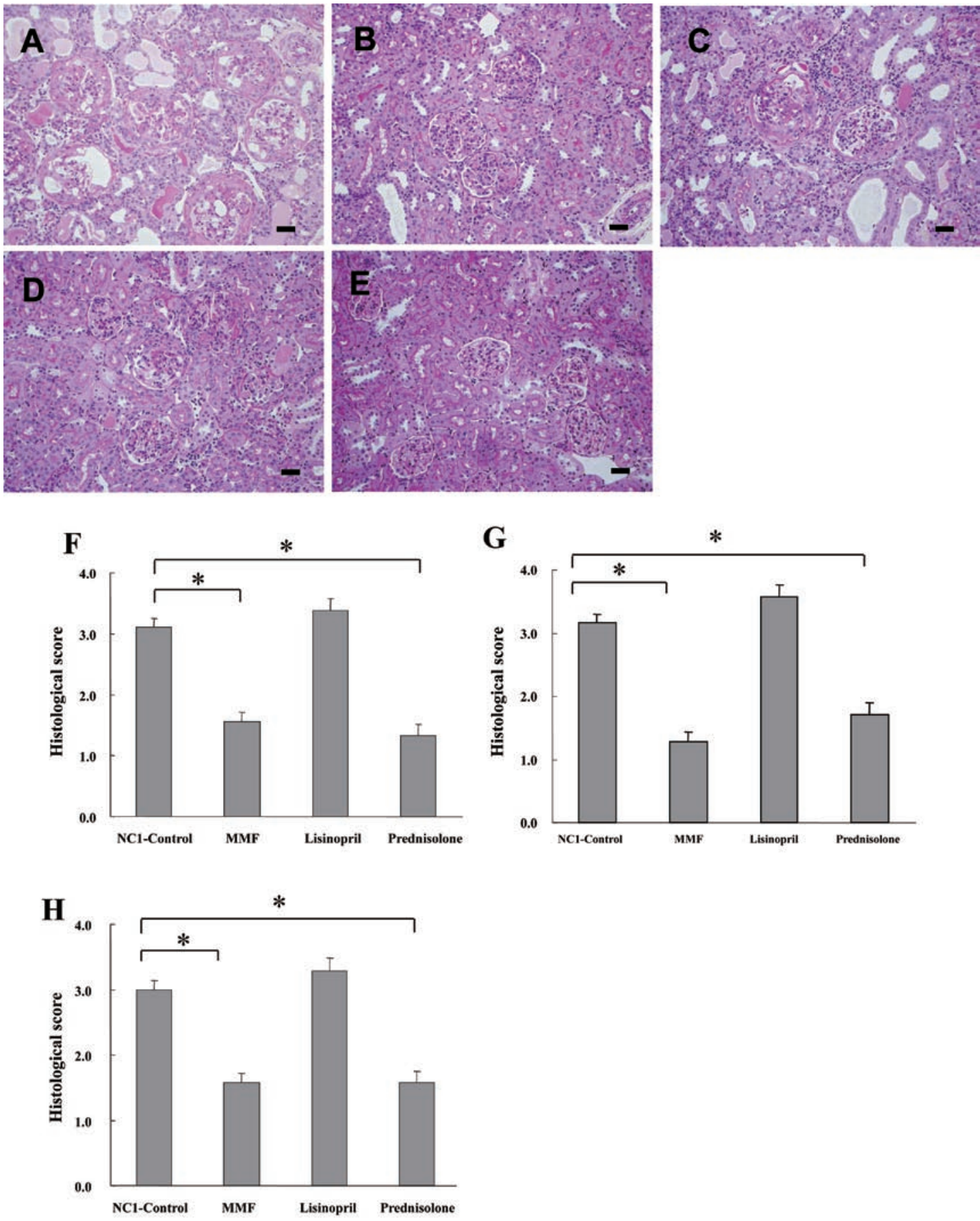

Fig. 9. Effects of prednisolone, MMF, and lisinopril on renal histopathology in WKY rats immunized with $\alpha 3$ (IV) NC1. Representative pictures are kidney sections of vehicle-treated control rats (A), MMF-treated rats (B), Lisinopril-treated rats (C), Prednisolone-treated rats (D), and normal rats (E) at day 35 after immunization with r $\alpha 3$ (IV)NC1. PAS-hematoxylin staining. Magnification $\times 200$. Bar $=50 \mu \mathrm{m}$. Histological analysis of crescent formation $(F)$, dilation of tubules $(G)$, and inflammation $(H)$ in WKY rats immunized with $\alpha 3(I V) N C 1$. Data represent means $\pm \mathrm{SD}$ of each group at Day $35 \mathrm{after}$ immunization with $20 \mu \mathrm{g}$ of r $\alpha 3$ (IV)NC1 in CFA. $* P<0.01$; comparison with the vehicle-treated control rats was performed by Student's $t$-test. 


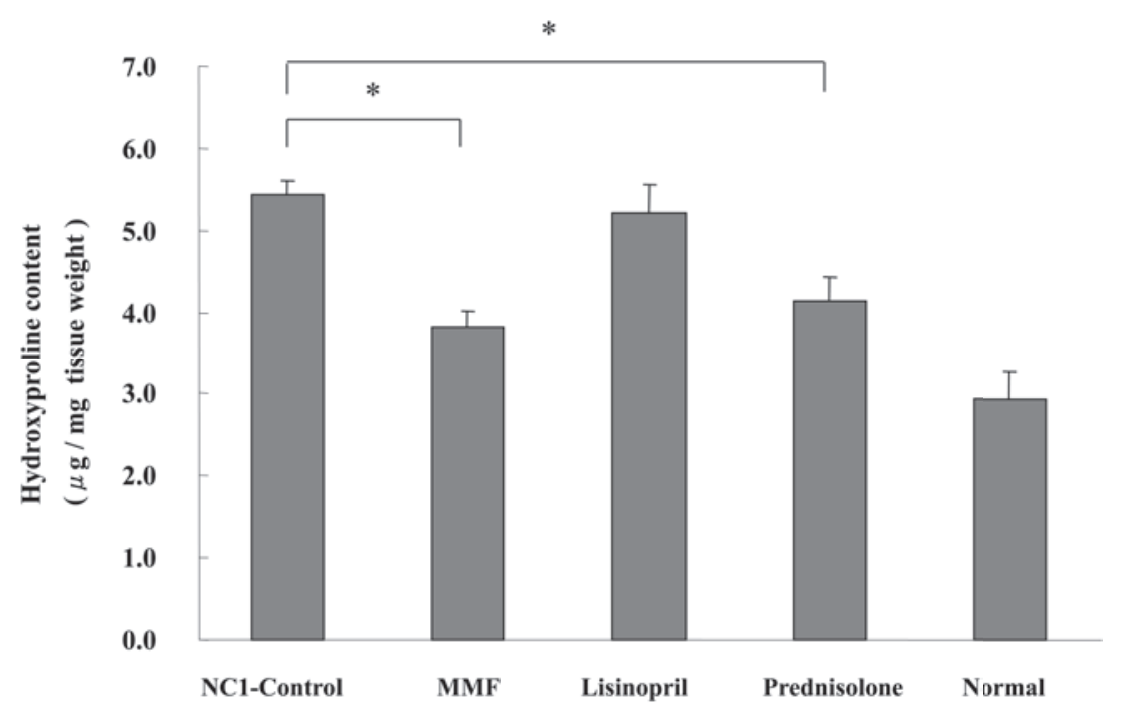

Fig. 10. Effects of MMF, lisinopril, and prednisolone on renal fibrosis with respect to hydroxyproline levels. Data represent means \pm SD of each group at Day 35 after immunization with $20 \mu \mathrm{g}$ of r $\alpha 3(\mathrm{IV}) \mathrm{NC} 1$ in CFA. ${ }^{*} P<0.01$; comparison with the vehicle-treated control rats was performed by Student's $t$-test.

\section{Discussion}

Several previous studies have demonstrated that passive transfer of anti- $\alpha 3$ (IV)NC1 antibodies or active immunization with purified $\alpha 3$ (IV)NC1 from several sources, or more recently, recombinant $\alpha 3$ (IV)NC1 induce glomerulonephritis in the rat $[8,11,23]$. However, in these nephritis models, the mechanisms of exacerbation of renal function has remained unclear and little attention has been paid to the progression to renal failure and renal fibrosis, critical pathological feature in glomerulonephritis.

In this study, we immunized WKY rats with recombinant $\alpha 3$ (IV)NC1 and successfully produced a rat glomerulonephritis model that resembles human glomerulonephritis in terms of the progression to renal failure and renal fibrosis. Using this animal model, we performed detailed clinicopathological studies to clarify the relationships between the dose of immunization antigen, and presence or absence of renal fibrosis and the histological nature of the lesion.

In the present study, we demonstrated for the first time that $\alpha 3$ (IV)NC1 antigen immunization alone of WKY rats is sufficient to induce renal dysfunction and that the development of renal dysfunction is closely associated with the dose of immunizing antigen. A single immunization of female WKY rats with a relatively low $(3 \mu \mathrm{g})$ dose of recombinant $\alpha 3$ (IV)NC1 antigen induced proteinuria but was not sufficient to induce renal dysfunction. In contrast, immunization with moderate $(10 \mu \mathrm{g})$ or relatively high $(20 \mu \mathrm{g})$ doses of $\alpha 3(\mathrm{IV}) \mathrm{NC} 1$ antigen induced not only proteinuria but also increased plasma creatinine and BUN. Furthermore, these rats showed significant histopathological features represented by severe crescent formation in glomeruli and renal fibrosis, as evidenced by the increase in hydroxyproline content. These findings therefore indicate that the dose of the immunizing antigen affects the level of histopathological changes in severe crescent formation, tubular injury and renal fibrosis and that these are associated with the increase of plasma creatinine and BUN.

Moreover, the results of the present study implicated dissociation between proteinuria and renal dysfunction. Proteinuria was not associated with histopathological changes and improvement of renal function in this model, implying that proteinuria and renal dysfunction may develop via different mechanisms.

In the serial study of urinalysis and renal biopsies, we found that circulating anti- $\alpha 3$ (IV)NC1 antibodies and proteinuria initially appeared, followed by an increase 
in plasma creatinine and BUN, and concomitantly, prominent renal fibrosis that was demonstrated by an increase in type I collagen and $\alpha$ SMA gene expression, and TGF- $\beta$ levels in the renal cortex. In other words, renal function rapidly worsened along with progression of renal fibrosis. TGF- $\beta$ is known to play an important role in extracellular matrix (ECM) metabolism. Sustained TGF- $\beta$ expression is responsible for the development of progressive renal fibrosis [30]. As expected, in the present study, the change of TGF- $\beta$ level was similar to that of hydroxyproline content in the renal cortex. The results of the present study indicate that expression of TGF- $\beta$ contributed to the excessive deposition of glomerular and tubulointerstitial ECM in this model and suggest that it has an important role in human glomerular disease as well. Furthermore, the present study revealed that up-regulation of type I collagen and $\alpha$ SMA is associated with renal dysfunction in this model. Type I collagen and $\alpha$ SMA are key participants in ECM turnover as well as TGF- $\beta$ and increase of these factors is known to be a hallmark of myofibroblast activation in the development of renal tubulointerstitial fibrosis [31]. RT-PCR analysis showed that significant up-regulation of type I collagen and $\alpha$ SMA gene expression were concomitant with deterioration of renal function (Fig. 7C). Therefore, we speculate that up-regulation of type I collagen and $\alpha$ SMA gene expression might be induced by immunization with $\alpha 3$ (IV)NC1 antigen, which leads to tubulointerstitial fibrosis in this model. In support of our findings, recent studies have demonstrated that the expression of $\alpha \mathrm{SMA}$, type I collagen, and TGF- $\beta$ increased in diseased kidneys and was accompanied by renal dysfunction, in other crescentic glomerulonephritis models induced by injection of anti-GBM antibody, and in models of Goodpasture's syndrome [3, 16, 17]. In addition, blocking of TGF- $\beta$ signaling inhibited crescent formation and glomerulosclerosis, prevented the development of fibrosis and improved renal function in a model of autoimmune crescentic glomerulonephritis [10]. Therefore these fibrogenic factors may play an important role in the development and progression of renal failure.

Below, we describe a speculative model of the mechanisms of development of end-stage renal failure in our model. Following immunization with a sufficient dose of $\mathrm{NC} 1$ antigen in female WKY rats, immunological events, including the binding of anti-NC1 antibody to GBM, initiate the development of proteinuria, crescent formation by rupture of the glomerular capillary wall and tubular injury. $\mathrm{NC} 1$ antigen immunization also induces myofibroblast activation, and expression of profibrotic cytokines such as TGF- $\beta$, followed by excessive deposition of ECM in the glomeruli and tubulointerstitium. This in turn provokes glomerulosclerosis and interstitial fibrosis, culminating in an irreversible stage, leading to renal dysfunction.

Immunosuppresive agents, prednisolone and MMF have reported therapeutic efficacy in treating rapidly progressive glomerulonephritis. MMF also has an antifibrosis effect in an immunologically induced glomerulonephritis as dose lisinopril, angiotensin-converting enzyme (ACE) inhibitor, in addition to its anti-inflammatory properties $[7,32]$. To characterize our model further, we investigated the effectiveness of these antinephritic agents which have different pharmacological properties. Treatment with prednisolone or MMF exerted significant suppressive effects in this model, improving renal function and all parameters of glomerular and interstitial injury. Moreover, these agents prevented renal fibrosis as detected by hydroxyproline content and both drugs were equally effective in preventing renal dysfunction and renal fibrosis in this model. In addition, no difference was observed when the efficacy of MMF was compared with that of prednisolone, suggesting that both drugs may act through a common pathway. We speculate that prednisolone and MMF inhibit glomerular and tubulointerstitial injury through immunological pathways. As a result, both drugs prevent renal dysfunction and progression of renal fibrosis before they reach an irreversible stage.

In contrast, lisinopril treatment prevented proteinuria and an increase in systolic blood pressure, partially, but not renal fibrosis and histological changes in this model. The preventive effect of lisinopril on renal fibrosis has been extensively studied in other animal models including the unilateral ureteral obstruction (UUO) model [7] and the remnant kidney model [2]. There is evidence that angiotensin II plays an important role in modulating inflammatory responses in the kidney. ACE inhibitors, including lisinopril, were shown to prevent macrophage 
recruitment and the proliferation of myofibroblasts in the remnant kidney model [29]. They also attenuate the progression of chronic renal disease through an anti-fibrotic effect that is largely dependent on changes in blood pressure $[9,14]$. However this effect was not observed in our model. Such a different efficacy between lisinopril and other drugs suggests that suppression of immune reaction-mediated glomerular and tubulointerstitial injury may be critical for the maintenance of renal function and for the prevention of progression of renal fibrosis in this model. The present findings therefore suggests that this model might be suitable for the evaluation of new therapeutic agents for treating rapidly progressive glomerulonephritis induced by immune reaction-mediated renal injury, such as anti-GBM glomerulonephritis, ANCA-associated glomerulonephritis and lupus nephritis.

In conclusion, we characterized the biochemical and histological features of a model of glomerulonephritis induced by $\alpha 3(\mathrm{IV}) \mathrm{NC} 1$. We demonstrated that immunization of female WKY rats could with a sufficient dose of recombinant $\alpha 3(\mathrm{IV}) \mathrm{NC} 1$ induces end-stage kidney disease with histology resembling that of human crescentic glomerulonephritis and tubulointerstitial fibrosis, that eventually progresses to renal failure. This model possesses reproducibility as a reliable disease model for renal failure.

Our model provides a useful tool for investigating pathogenetic mechanisms and researching new therapeutic approaches for human crescentic glomerulonephritis accompanied with immune reaction-mediated renal injury and renal fibrosis.

\section{Acknowledgments}

This work was presented in part at the 36th Annual meeting of the American Society of Nephrology, St. Louis in November 2004. We express our gratitude to Professor. Toshio Doi of the University of Tokushima, Japan, for his kind advice.

\section{References}

1. Abbate, M., Kalluri, R., Corna, D., Yamaguchi, N., McCluskey, R.T., Hudson, B.G., Andres, G., Zoja, C., and Remuzzi, G. 1998. Experimental Goodpasture's syndrome in Wistar-Kyoto rats immunized with $\alpha 3$ chain of type IV collagen. Kidney Int. 54: 1550-1561.

2. Abbate, M., Zoja, C., Rottoli, D., Corna, D., Tomasoni, S., and Remuzzi, S. 2002. Proximal tubular cells promote fibrogenesis by TGF- $\beta 1$-mediated induction of peritubular myofibroblasts. Kidney Int. 61: 2066-2077.

3. Akimoto, H. 2000. The possible role of adhesion molecule alpha 3 integrin, in the synthesis of intracrescentic extracellular matrix in accelerated anti-GBM nephritis. Nippon Jinzo Gakkai Shi 42: 1-10 (in Japanese).

4. Butkowski, R.J., Langeveld, J.P., and Wieslander J.P. 1987. Localization of the Goodpasture epitope to a novel chain of collagen IV. J. Biol. Chem. 262: 7874-7877.

5. Couser, W.G. 1999. Sensitized cells come of age: a new era in renal immunology with important therapeutic implications. J. Am. Soc. Nephrol. 10: 664-665.

6. Fujii, T., Watanabe, F., and Okuda, J. 1977. Rinsyokensa manual. pp. 251-259. Rinsho Kagaku Souron, Hirokawa Shoten, Tokyo (in Japanese).

7. Gonçalves, R.G., Biato, M.A., Colosimo, R.D.G., Martinusso, C.A., Pecly, I.D., Farias, E.K., Cardoso, L.R., Takiya, C.M., Ornellas, J.F.R., and Leite, M. Jr. 2004. Effects of mycophenolate mofetil and lisinopril on collagen deposition in unilateral ureteral obstruction in rats. Am.J. Nephrol. 24: 527-536.

8. Hopfer, H., Maron, R., Butzmann, U., Helmchen, U., Weiner, H.L., and Kalluri, R. 2003. The importance of cell-mediated immunity in the course and severity of autoimmune antiglomerular basement membrane disease in mice. FASEB $J$. 17: 860-868.

9. Ishidoya, S., Morrissey, J., McCracken, R., Reyes, A., and Klahr, S. 1995. Angiotensin II receptor antagonist ameliorates renal tubulointerstitial fibrosis caused by unilateral ureteral obstruction. Kidney Int. 47: 1285-1294.

10. Ka, S.M., Huang, X.R., Lan, H.Y., Tsai, P.Y., Yang, S.M., Shui, H.A., and Chen, A. 2007. Smad7 gene therapy ameliorates an autoimmune crescentic glomerulonephritis in mice. Am. J. Nephrol. 18: 1777-1788.

11. Kalluri, R., Danoff, T.M., Okada, H., and Neilson, E.G. 1997. Susceptibility to anti-glomerular basement membrane disease and Goodpasture syndrome is linked to MHC Class II genes and the emergence of T cell-mediated immunity in mice. J. Clin. Invest . 9: 2263-2275.

12. Kalwasser, H. and Schlegel, H.G. 1966. NADH-dependent coupled enzyme assay for urease and other ammoniaproducing systems. Anal. Biochem. 16: 132-138.

13. Kanno, K., Okumura, F., Toriumi, W., Ishiyama, N., Nishiyama, S., and Naito, K. 1998. Nephrotoxic seruminduced nephritis in Wistar-Kyoto rats: a model to evaluate antinephritic agents. Jpn. J. Pharmacol. 77: 129-135.

14. Klahr, S. and Morrissery, J. 1998. Angiotensin II and gene expression in the kidney. Am. J. Kidney 31: 171-176.

15. Neilson, E.G., Kalluri, R., and Sun, M.J. 1993. Specificity of Goodpasture autoantibodies for the recombinant noncollagenous domains of human type IV collagen. J. Biol. Chem. 268: 8402-8405.

16. Ng, Y.Y., Chen, Y.M., Tsai, T.J., Lan, X.R., Yang, W.C., and Lan, H.Y. 2009. Pentoxifylline inhibits transforming growth 
factor-beta signaling and renal fibrosis in experimental crescentic glomerulonephritis in rats. Am. J. Nephrol. 29: 43-53.

17. Okada, H., Inoue, T., Kanno, Y., Kobayashi, T., Ban, S., Kalluri, R., and Suzuki, H. 2001. Renal fibroblast-like cells in Goodpasture syndrome rats. Kidney Int. 60: 597-606.

18. Pusey, C.D. and Rees, A.J. (Eds). 1998. Rapidly Progressive Glomerulonephritis, Oxford University Press, Oxford.

19. Reynolds, J., Albouainain, A., Duda, M.A., Evans, D.J., and Pusey, C.D. 2006. Strain susceptibility to active induction and passive transfer of experimental autoimmune glomerulonephritis in the rat. Nephrol.Dial. Transplant. 21: 3398-3408.

20. Reynolds, J., Moss, J., Duda, M.A., Smith, J., Karkar, A.M., Macherla, V., Shore, I., Evans, D.J., Woodrow, D.F., and Pusey, C.D. 2003. The evolution of crescentic nephritis and alveolar haemorrhage following induction of autoimmunity to glomerular basement membrane in an experimental model of Goodpasture's disease. J. Pathol. 200: 118-129.

21. Ryan, J.J., Katbamna, I., Mason, P.J., Pusey, C.D., and Turner, A.N. 1998. Sequence analysis of the 'Goodpasture antigen' of mammals. Nephrol. Dial. Transplant. 13: 602607.

22. Ryan, J.J., Reynolds, J., Norgan, V.A., and Pusey, C.D. 2001. Expression and characterization of recombinant rat $\alpha 3$ (IV) NC1 and its use in induction of experimental autoimmune glomerulonephritis. Nephrol. Dial. Transplant. 16: 253261.

23. Sado, Y., Boutaud, A., Kagawa, M., Naito, I., Ninomiya, Y., and Hudson, B.G. 1998. Induction of anti-GBM nephritis in rats by recombinant $\alpha 3$ (IV)NC1 and $\alpha 4$ (IV)NC1 of type IV collagen. Kidney Int. 53: 664-671.

24. Saus, J., Wieslander, J.P., and Langeveld, J.P. 1988. Identification of the Goodpasture antigen as the alpha 3 (IV) chain of collagen IV. J. Biol. Chem. 263: 13374-13380.

25. Stanton, M.C. and Tange, J.D. 1958. Goodpasture's syndrome (Pulmonary hemorrhage associated with glomerulonephritis). Aust. Ann. Med. 7: 132-144.

26. Stegemann, H. and Stalder, K. 1967. Determination of hydroxyproline. Clin. Chim. Acta 18: 267-273.

27. Tipping, P.G. and Holdsworth, S.R. 2006. T cells in crescentic glomerulonephritis. J. Am. Soc. Nephrol. 17: 1253-1263.

28. Wilson, C.B. and Dixon, F.J. 1973. Anti-glomerular basement membrane antibody-induced glomerulonephritis. Kidney Int. 3: 74-89.

29. Wu, L.L., Yang, N., Roe, C.J., Cooper, M.E., Gilbert, R.E., Atkins, R.C., and Lan, H.Y. 1997. Macrophage and myofibroblast proliferation in remnant kidney: role of angiotensin II. Kidney Int. 63: S221-225.

30. Yamamoto, T., Nobel, N.A., Cohen, A.H., Nast, C.C., Hishida, A., and Gold, L.I. 1994. Expression of transforming growth factor- beta isoforms in human glomerular disease. Kidney Int. 45: 486-497.

31. Yang, J. and Liu, Y. 2001. Dissection of key events in tubular epithelial to myofibroblast transition and its implications in renal interstitial fibrosis. Am. J. Pathol. 159: 1465-1475.

32. Zang, C., Zhu, Z., Wang, G., and Deng, A. 2003. Effecs of mycophenolate mofetil on renal interstitial fibrosis after unilateral ureteral obstruction in rats. J. Huazhong Univ. Sci. Technol. Med. Sci. 23: 269-270. 